\title{
Ein Kaufmann aus Naukratis
}

Im ägyptischen Museum in Kairo gibt es unter den Denkmälern der Spätzeit seit langer Zeit ein in mancherlei Hinsicht bemerkenswertes Stück ${ }^{1}$, das aber bislang wenig Beachtung gefunden hat. Es ist aus sehr fein geflecktem rotbraunem Granit und sieht auf den ersten Blick aus wie eine Glocke, die auf einem quadratischen Sockel steht. Das ganze Objekt (aus einem Stück gearbeitet) ist $50 \mathrm{~cm}$ hoch, die Basis hat eine Kantenlänge von jeweils $53 \mathrm{~cm}$ und eine Höhe von $14 \mathrm{~cm}$. Auf dieser Basis steht ein glockenförmiger Gegenstand, dessen Durchmesser unten $51 \mathrm{~cm}$ beträgt. Oben ist er durch eine Art kreisförmigen „Aufsatz“ mit senkrechten Seiten begrenzt, der $8,5 \mathrm{~cm}$ hoch ist und einen Durchmesser von $27 \mathrm{~cm}$ hat. In der Oberseite dieses ringförmigen oberen Abschlusses ist ein kreisrundes Loch von $12 \mathrm{~cm}$ Durchmesser und ca. 2,5 cm Tiefe eingeschnitten, dessen Boden nicht richtig geglättet worden ist. Offenbar hat der ganze Gegenstand als eine Art Untersatz gedient, wohl für einen Altar o. ä. Der Form nach wäre es denkbar, daß es sich ursprünglich um ein Säulenkapitell mit Abakus handelte, das dann auf den Kopf gestellt - zweckentfremdet worden wäre ${ }^{2}$. Wie auch immer, zumindest seit die In-

Derzeitiger Standort: R 30 centre (E). Ich danke dem Direktor des Ägyptischen Museums Kairo, Dr. M. Saleh, sehr herzlich für die Genehmigung zur Publikation und zur Anfertigung der Aufnahmen (alle Fotos: Museum Kairo).

Möglicherweise hat schon Maspero (s. u., Fußnote 4) etwas Ähnliches vermutet; wenigstens hält er es für möglich, daß die Inschrift sekundär ist. 


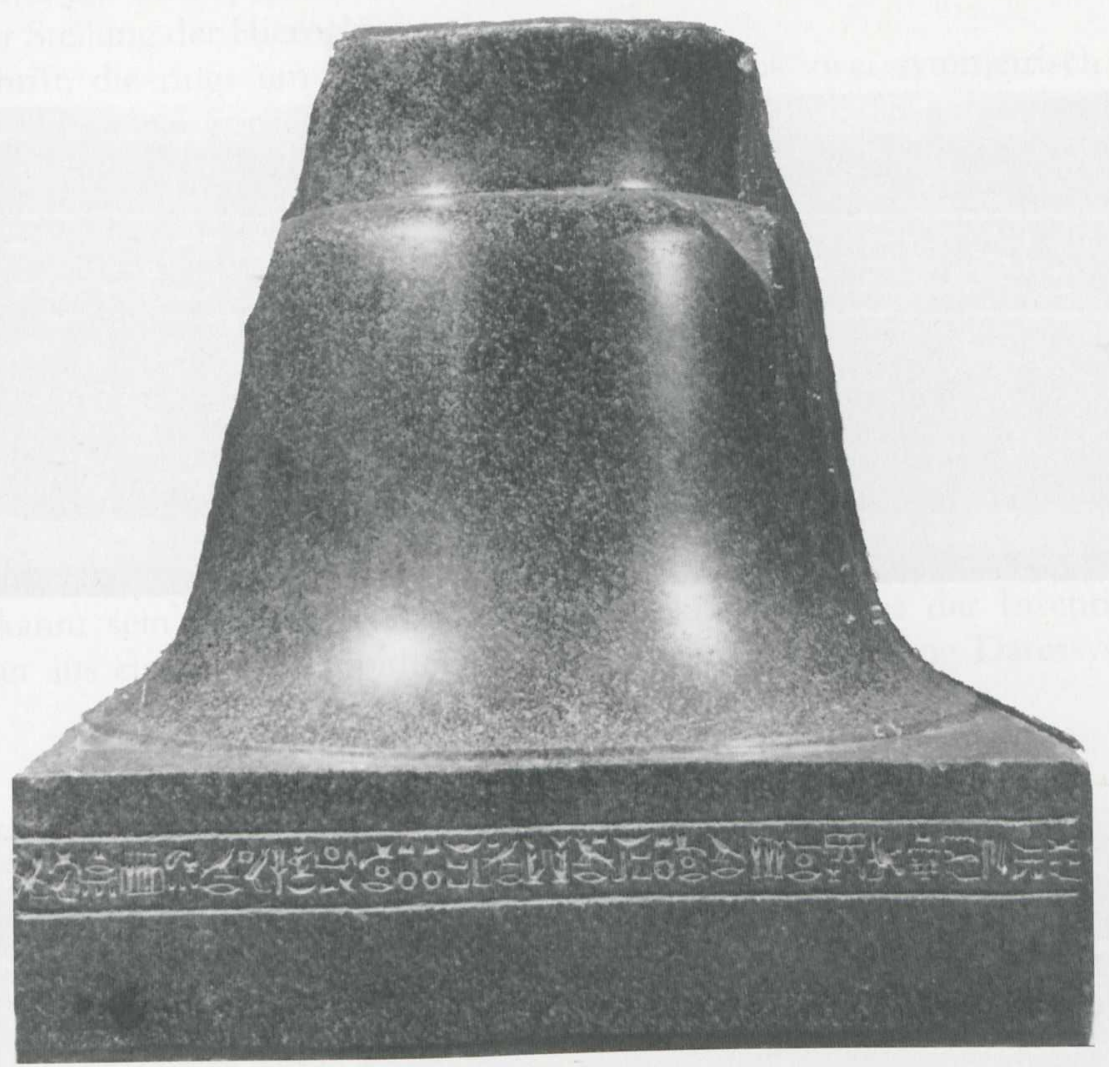

a

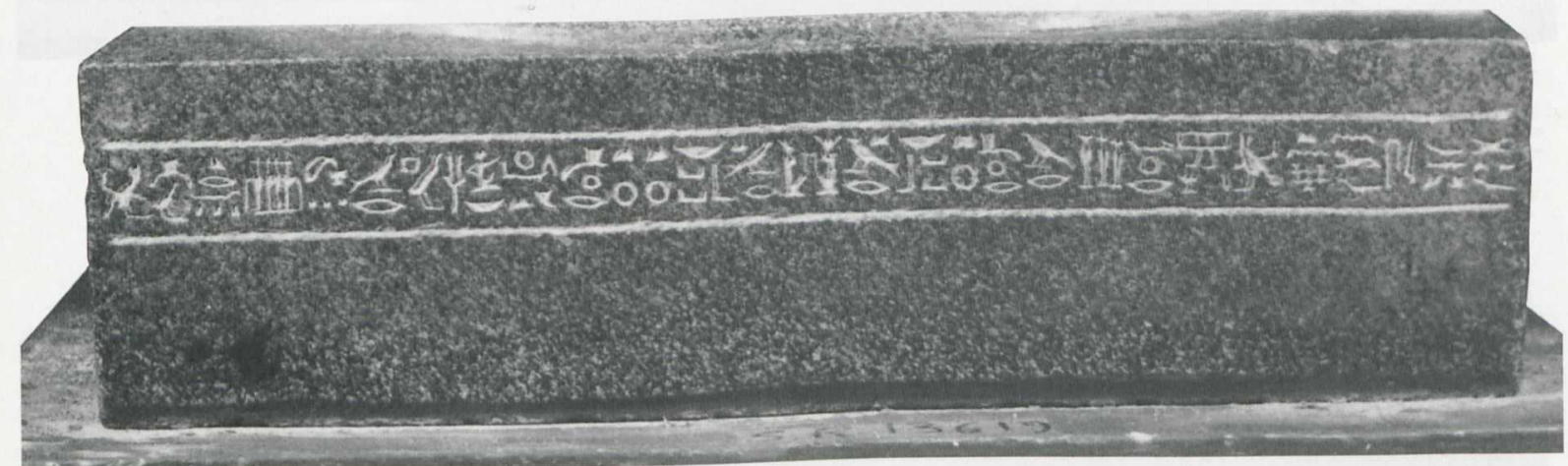




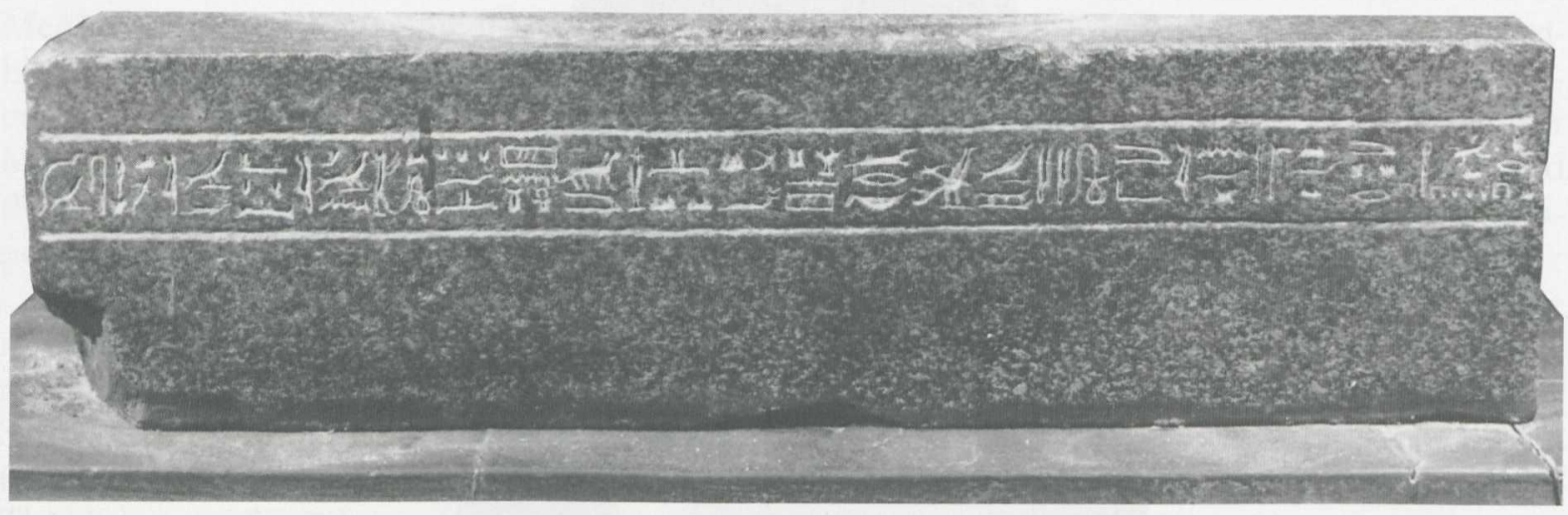

a
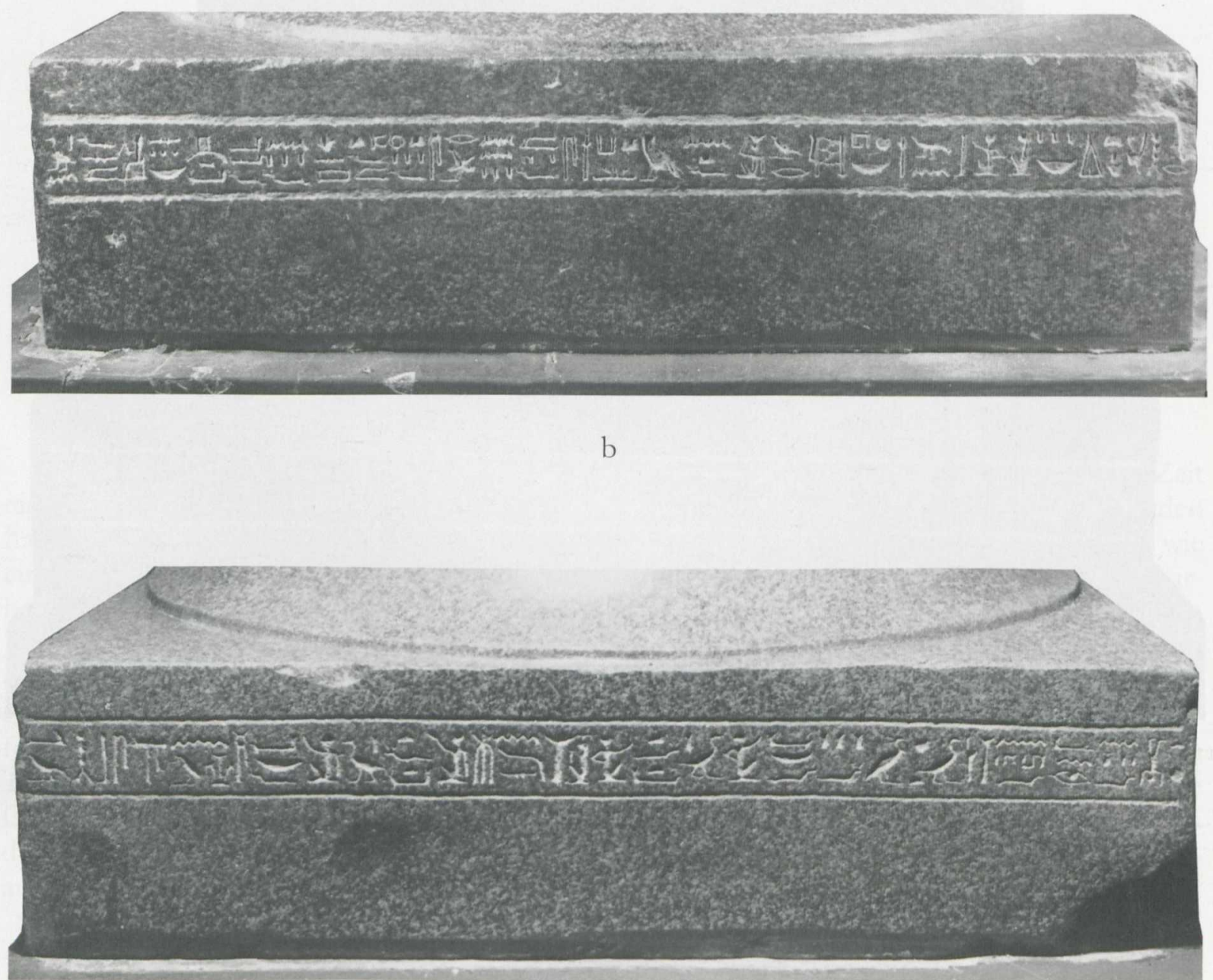
schrift eingeschnitten wurde, diente das Stück als Untersatz, mit dem quadratischen Teil als Sokkel, wie aus der Stellung der Hieroglyphen hervorgeht.

Diese Inschrift, die rings um die Basis läuft, besteht aus zwei symmetrisch angebrachten Texten, in der Mitte einer Sockelseite beginnend und in der Mitte der gegenüberliegenden Seite endend.

Die Inschrift ist 1900 von G. Daressy in seinen „Notes et remarques“ publiziert worden ${ }^{3}$. Ansonsten wird das Stück in PM IV, 50 erwähnt sowie in einigen Museumskatalogen ${ }^{4}$. In den Inventarbüchern des Museums ist es im Jahr 1924 unter der vorläufigen Nummer 1/6/24/6 registriert $^{5}$ worden und wird dort (nach Masperos Katalog) als „,base d'autel en forme de cloche renversée" beschrieben. Es muß aber einige Zeit vor dem Jahre 1900 ins Museum gelangt sein: Im Wörterbuch-Archiv in Berlin befindet sich neben einem Abklatsch der Inschrift durch Sethe aus dem Jahr 1905 ein weiterer, den H. Brugsch angefertigt hat, und das muß vor 1892 gewesen $\operatorname{sein}^{6}$.

Über Fundort und Fundumstände sagen das Inventarbuch und die Publikationen nichts, sie dürften unbekannt sein?. Die Herkunftsangabe „Naukratis“ ist aus der Inschrift erschlossen (genauer gesagt aus einem Götterepitheton), wie aus der Formulierung Daressys deutlich hervorgeht .

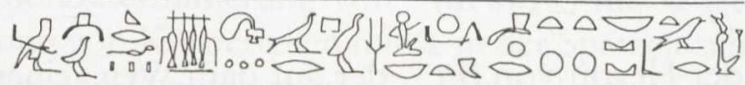

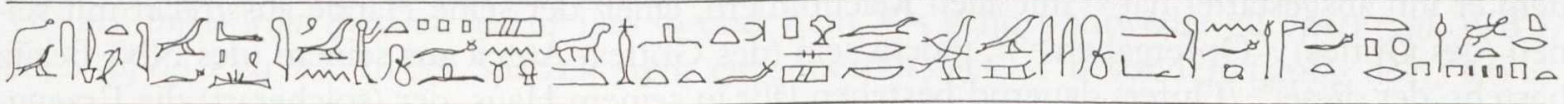

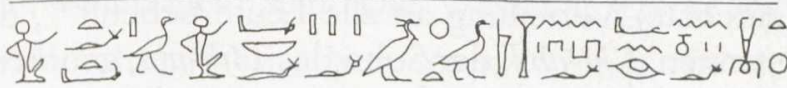

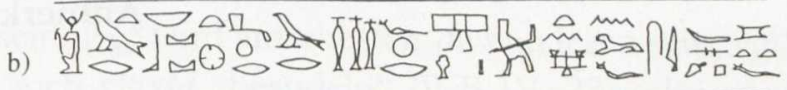

दून

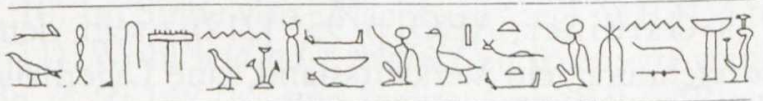

Rec'Trav 22, 140-141 (CLXXI).

Z. B. in G. Maspero, Guide to the Cairo Museum, Kairo 1908, p. 214 (663); id., Guide du visiteur au Musée du Caire, Kairo 1915, p. 207 (852).

Sonstige Nummern: S.R. 13619; Guide-Nr. 852.

Vgl. S. Köpstein, in: Mitteilungen aus der Arbeit am Wörterbuch der ägyptischen Sprache, 3 (1994), 16.

Der oben erwähnte Katalog von 1908 gibt allerdings „Damanhur“ an.

Op. cit., p. 140. Vgl. J. Yoy otte, RdE 34, 1982/83, 136 „Daressy concluait à juste titre qu'il fallait ranger cette pièce, parmi les monuments égyptiens provenant de Naucratis" ". 
Daressy hat das Objekt aufgrund des Namens seines Besitzers, Nht-nb.f, in die frühe Ptolemäerzeit gesetzt ${ }^{9}$, und in PM $(I V, 50)$ hatte man sich dem angeschlossen.

Obwohl die Inschrift schon 1900 veröffentlicht wurde und ägyptische Texte aus Naukratis sehr rar sind, ist $\mathrm{m}$. W. nie eine Übersetzung erschienen. Das hängt sicherlich nicht zuletzt damit zusammen, daß der Text recht schwierig ist; die folgende Übersetzung stellt auch nur einen ersten Versuch dar. Wie unsicher sie ist, ergibt sich schon aus der ungebührlichen Zahl von Emendationen, die mir nötig erschienen ${ }^{10}$.

\section{Übersetzung"}

a) „Der Ehrwürdige bei Mut, der Großen, der Herrin von Btt ${ }^{(1)}$, der Reiche, der Herr von Besitztümern $^{(2)}$, mit vielen Schätzen und wertvollen Beuteln, mit weiten Magazinen ${ }^{(3)}$ und zahlreichen Schatzhäusern, der geworden ist, wie Gott ihm das Schicksal befohlen hat ${ }^{(4)}$, frei von Räuberei $^{(5)}$, der nicht gegen einen anderen wütet wegen seines Anteils; der nicht umhergeht mit seinen Geschenken ${ }^{(6)}$, während (andrerseits) der Hablose nicht seinen Tadel erfleht ${ }^{(7)}$, ein Gerechter, der es verabscheut, Verleumdungen weiterzugeben ${ }^{(8)}$, der sich zu seinem Nächsten herabläßt ${ }^{(9)}$, freundlich zu seinem Viertel ${ }^{(10)}$, von glücklichem Charakter gegenüber seinen Mitbürgern ${ }^{(11)}$, Nht-nb.f, der Sohn des T3j:f-nht. “"

b) „Der Ehrwürdige bei Hathor, der Großen, der Herrin von $B t t^{(1)}$, der auf dem Weg seines Herrn geht, von großer Gunst bei ihm ${ }^{(12)}$, der ihn ausgezeichnet hat vor seinesgleichen ${ }^{(13)}$, nachdem er ihn ausgestattet hat ${ }^{(14)}$ mit allen Reichtümern, einer, der seine Hände ausstreckt mit seinen (des Gottes) <Opfergaben> ${ }^{(15)}$, indem sein (des Gottes) Anteil aus seinem (des NN) Besitz besteht, der diese ${ }^{(16)}$ (Opfer) dauernd bestehen läßt in seinem Haus, der (solcherart) die Erzeugnisse ${ }^{(17)}$ vermehrt durch das, was herbeigetragen wird ${ }^{(18)}$, mit großen Opfergaben täglich, der die (gerechte) Verteilung an alle Leute befiehlt ${ }^{(19)}$, der Brot gibt dem Hungrigen und Kleider dem Nackten, Nht-nb.f, der Sohn des T3j.f-nht, geboren von der Ns-Nbt-hwt."

\section{Anmerkungen}

1) Zum Ortsnamen Btt und seinen Schreibungen vgl. J. Yoyotte, RdE 34, 1982/83, $129-36$.

2) Trotz des Determinativs handelt es sich zweifellos um das Wort `h` $w$ „Haufen“, vgl. WB I, 220, 14. Das Determinativ könnte aber darauf hindeuten, daß hier eine Ortsbezeichnung (Schatzhaus o. ä.) gemeint ist (Vorschlag J. Osing).

3) In diesem Zusammenhang kann $\frac{\pi}{n} \frac{\Delta}{a}$ eigentlich nur eine Ableitung von dem im NR belegten semitischen Fremdwort $m k r$ „Kaufmann“ (s. Gardiner, AEO, p. 95* [212]) ${ }^{12}$ sein, dem Determinativ zufolge eine Gebäude- oder Ortsbezeichnung, ein Verkaufsstand, eine Lagerhalle oder etwas ähnliches. Eine Verbindung mit mqrt „(Augen)Höhle“ (Gardiner, RdE 11, 1957,

\footnotetext{
Maspero in den genannten Katalogen denkt dagegen an die Perserzeit.

${ }^{10}$ Wenn auch der Text zumindest an einigen Stellen unzweifelhaft fehlerhaft ist, z. B. bei der Schreibung von psšt mit $\bigcirc$ statt $\square$ oder bei $\Theta \square$ für hntj.

${ }^{1}$ Ich danke J. Osing herzlich für eine Durchsicht dieses Übersetzungsversuchs. Seine Verbesserungen und Änderungsvorschläge sind an den entsprechenden Stellen vermerkt worden.

${ }^{12}$ Vgl. auch J. E. Hoch, Semitic Words in Egyptian Texts of the New Kingdom and the Third Intermediate Period, Princeton 1994, p. 169 (223).
} 
50, n. 2; pl. 3, Z. 62) bzw. mqr (ein Gefäß, s. C. Evrard-Derriks/J. Quaegebeur, CdE 54, 1979, 46-9) (Vorschlag J. Osing), beides vielleicht so etwas wie Ausgehöhltes bedeutend, scheint mir weniger wahrscheinlich.

4) Das Verständnis der Phrase hinter $p r-\underline{h} \underline{d}$ als $m$ hpr $h f t w \underline{d} . n . f n t r r s(w)$ ist sehr fraglich. Übersetzt man so, wäre $\frac{0}{\sigma}$ in $\frac{0}{\sigma}$ zu emendieren; die Inschrift enthält allerdings auch sonst einige Fehler, s. o. $\bigcirc$ ist zweifellos $₫ 3(w)$ zu lesen (Hinweis J. Osing); zu der Verbindung $w \underline{d} \varsigma_{3}$ vgl. u. a. J. Quaegebeur, Le dieu égyptien Shaï dans la religion et l'onomastique, OLA 2, 1975, p. 93; 103-4.

5) Die beiden letzten Phrasen sollen wohl bedeuten, daß er durch Gottes Hilfe reich geworden ist und eben nicht durch unrechtmäßige Machenschaften.

6) 不

7) Das $f$ von $\underline{t z w} . f$ ist natürlich genetivus obiectivus („das ihn Tadeln“). Diese Übersetzung der Phrase bedingt, daß das kleine Zeichen über dem ein (schlecht geschnittenes) 9 ist und das horizontale Zeichen unter šwj-` ein $₫(t z)$. Es wäre dann $n t w 3 . n$ šwj-` $t z w . f$ zu lesen. Statt eines 9 könnte allerdings auch $\bigcirc(f)$ zu lesen sein (man beachte aber das Determinativ $Q$ von ?, das eine ganz andere Form hat!) und das $\gg$ als $w$ (Vorschlag J. Osing): Die drei Striche sprechen mehr für die Buchrolle, die beidseitig konkave Form eher für $\underline{t z}$. Dann müßte man übersetzen , indem er (andrerseits) nicht bettelt (o. ä.) wegen seiner Hablosigkeit“" (also $n$ tw3.f $n$ šwj- $f$ ). Die Determinierung von ` (in $\check{w} w j-$ ) mit $Q$ ist ungewöhnlich. Generell ist aber die Determinierung von Körperteilen mit $Q$ in der Spätzeit sehr viel häufiger als in älteren Inschriften. Die beiden Phrasen jwtj phr $m$ šnw.f und $n$ tw $3 . n \varsigma w j-\ulcorner t z w . f$ gehören wohl zusammen und bedeuten, daß er einerseits nicht mit seinem Reichtum protzte, andrerseits nicht geizig gegenüber Bedürftigen war.

8) $m 3^{c} b w(t) w h m \underline{d} w s$ zu lesen? Das $\square$ über dem gehört entweder zu $b w(t)$ (vgl. WB I, $453,5-6)$ oder zu $m 3^{\varsigma}$ : In der Spätzeit steht ein ,überflüssiges“ . $t$ besonders häufig dort, wo es ein oft gebrauchtes feminines Wort derselben Wurzel gibt $\left.(m)^{c} t\right)$. 古 (so deutlich, Daressy hat könnte eine Schreibung von whm ,wiederholen, weitersagen“ sein. Die Wiedergabe von $\mathrm{nm}$ durch fo u. ä. ist gut dokumentiert, vgl. H. G. Fischer, Varia, New York 1976, p. 101-4; F. Daumas, Valeurs phonétiques des signes hiéroglyphiques d'époque Gréco-Romaine, Montpellier 1988, p. 269. Die umgekehrte Verwendung von für whm könnte auf eine in der Spätzeit so häufige „Hyperkorrektheit“ zurückzuführen sein. $\int_{1}^{2}$ wird am ehesten $\underline{d} w s$ zu lesen sein, vgl. WB V, 552, 6. Nicht auszuschließen wäre aber auch s3t/s3d „,besudeln“ (WB IV, 27) oder $s \underline{d w j}$ „schlechtmachen“.

9) Zu $d h(n) n$ snw.f vgl. WB V, 485, 1 und meine Ägyptischen Biographien der 22. und 23. Dynastie, Wiesbaden 1985, p. 60 (79), wo auch diese Stelle (nach Daressy) zitiert wird. Ein weiterer Beleg in ziemlich unklarem Kontext auf der unpublizierten Statue Kairo JE $37149^{13}$, rechts, Z. 6: (文舟 genden[?],) dh n sn.nw.f (der den Agressor mit allen seinen Mitteln [?] beruhigt)".

10) Im Sinne von „Nachbarn“, vgl. WB II, 110, 8-9.

11) Wohl w3d qd $r$ nwtjw.f zu lesen; zu vergleichbaren Phrasen s. J. J. Clère, RdE 6, 1950, 138, Z. 2; $142(\bar{K})$ und meine Ägyptischen Biographien, p. 116-7 (11). Möglich wäre natürlich auch, (w3 $\underline{d}$ qd) htm nwtjw.f, ,der seine Mitbürger ausstattet“" zu lesen (Vorschlag J. Osing).

12) Das erste Beiwort dieses Abschnitts wird durch jm3hh(w) hr Hwt-Hrw wrt nb(t) Btt und šm $h r m t n n n b . f$ eindeutig abgegrenzt. Es dürfte $w r h z w t h r . f$ zu lesen sein, mit ganz ungewöhnlicher „Voranstellung aus Respekt" des Suffixes. Dieses Suffix hängt allerdings in der Luft, denn es hat kein vorhergehendes Bezugswort: Der Gottesname in der jm3hw-Formel ist ja eindeutig feminin. Es sieht so aus, als seien die ersten beiden Epitheta vertauscht worden, und eine Um-

Aus der Cachette von Karnak, Nr. K 458; derzeitiger Standort R 24 W4, unten. 
stellung ergibt auch inhaltlich eine sinnvollere Abfolge: Seinem Herrn ergeben - von ihm begünstigt (generell) - von ihm ausgezeichnet vor anderen. Bei diesem Herrn dürfte es sich, wie aus dem Folgenden hervorgeht, um einen Gott handeln ${ }^{14}$, kaum um den König. Eine andere Möglichkeit, die eine geringfügigere Emendation erfordern würde, wäre es, das erste Beiwort wr $h$ hwt.f $h r<. s>\mathrm{zu}$ lesen, als parenthetischen Einschub ,groß ist seine Gunst bei <ihr>“ (Vorschlag E. Blumenthal).

13) sth sw hntj mjtj(w).f.

14) htm.n.fsw.

15) Die Gruppe ${ }^{1|| \mid}$ scheint zunächst wenig Sinn zu machen. Die Übersetzung beruht auf

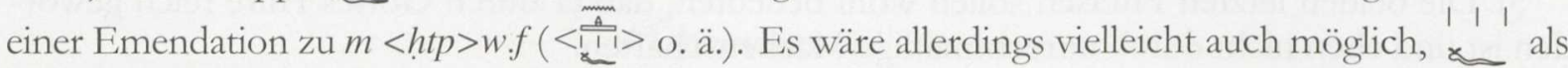
hmtw. $f$,,seine Gefährten“"zu lesen (vgl. EAG, $\int 409$; Sethe, Zahlworte, p. 120: Vorschlag J. Osing); dann ergäbe sich $3 w j$ ' $w w j . f n$ hmtw.f pšs.f $m$ ht.f ,der seine Hände ausstreckt seinen Gefährten, indem er austeilt von(?) seinem Besitz". Das hätte den großen Vorzug, keine Textemendation zu erfordern. Allerdings wäre die Schreibung doch sehr knapp, und das Wort scheint bisher nur in ganz alter Zeit belegt zu sein. Zudem würde es wohl nicht so gut in den Zusammenhang passen.

16) Schreibungen von $n n$ mit Pluralstrichen kennt das WB nicht, sie sind aber vereinzelt belegt,

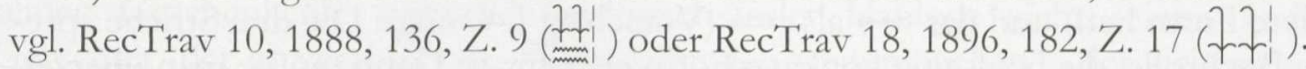

17) Vgl. WB V, 100, 1-2.

18) $n t j f 3 j(w)$ ? Die Deutung auch dieser letzten Phrase ist unsicher, die Schreibung wäre ungewöhnlich. Falls sie so zu verstehen ist, müßte die gesamte Phrase bedeuten, daß Nht-nb.f seinen Besitz (oder jedenfalls einen Teil davon) dem Gott zur Verfügung stellte, also zu den Erträgen der tempeleigenen Güter zusätzliche Lieferungen stiftete.

Eine ganz andere Möglichkeit wäre es, $k 3 t m$ zu $m k 3 t$ zu emendieren: Dann ergäbe sich „(. . . in seinem Haus,) das vergrößert worden ist durch (Bau)Arbeiten für seinen Vater". Die erste Möglichkeit ergibt aber wohl einen kohärenteren Text.

19) Der also die (von ihm gespendeten) Umlaufopfer gerecht verteilen läßt. Zu sšm(w) vgl. T. E. Peet, The Rhind Mathematical Papyrus, London 1923, p. 22, n. 1; P. Posener-Kriéger, Les Archives du temple funéraire de Néferirkarê-Kakaï, BdE 65, 1976, p. 7; 338c; 220 -1 (mit weiteren Angaben); vgl. auch Goedicke, MDAIK 21, 1966, 37.

Aus der Inschrift geht zunächst klar hervor, daß es sich bei dem Stifter um einen sehr reichen Mann gehandelt hat, und zwar um einen Kaufmann, wie zumindest ein Beiwort (3wj mkrt, s. o., Anm. 3) deutlich macht. Diese Betonung des Reichtums ist für ägyptische biographische Texte zumindest ungewöhnlich. Es kommen zwar auch sonst vereinzelt Phrasen in diesem Sinn vor ${ }^{15}$, aber nie in derartiger Ausschließlichkeit, ich kenne jedenfalls nichts Vergleichbares. Gleichfalls sehr ungewohnt ist die Tatsache, daß der Mann keinen einzigen Titel führt, nicht einmal einen Priestertitel, obwohl er doch beansprucht, die Verteilung der Opfer bestimmt zu haben.

Die Namen des Besitzers (Nht-nb.f), seines Vaters (T3j.f-nht) und seiner Mutter (bzw. Großmutter, s. u.: Ns-Nbt-hwt) sind alle gut ägyptisch. Dennoch wäre es natürlich grundsätzlich möglich, daß es sich um einen Griechen handelte. Man vergleiche die Statue Kairo CG $1230^{16}$ aus Naukratis, die einem Mann mit dem ägyptischen Namen $H r w-m-h 3 b$ gehört, der sich selbst aber als Griechen $(h 3 w-n b w t)$ bezeichnet.

Vielleicht Amun von Naukratis, vgl. dazu Yoyotte, RdE 34, 1982/83, 129-36.

Z. B. auf einem Torso in Alexandria (s. J. Vercoutter, BIFAO 49, 1950, 103; pl. V, Z. 2), dem Naophor Kairo CG 689 (Rückenpfeiler, Z. 3, s. P. Montet, Kêmi 7, 1938, 134-5), der Schreiberstatue Kairo CG 691 (Rückenpfeiler Z. 2, s. L. Borchardt, Statuen und Statuetten, III, 36), auf dem Sarg Kairo CG 29301 (s. G. Maspero, Sarcophages des époques persane et ptolémaïque, I, 10, Z. 2), im Grab des Petosiris (Inschriften Nr. 58, b; c; 100 , s. G. Lef èbvre, Le tombeau de Petosiris, II, 29-30; 30-1; 73-4; I, 86-7; 88-9; 183-4), im Grab des Schoschenk (TT 27, s. A. Roccati, OrAnt 12, 1973, Fig. 1, Z. 15-6; vgl. Merikare, 61!) u. a. m.

${ }^{16}$ Borchardt, Statuen und Statuetten, IV, 120-1. 
Vielleicht ist von dem Vater des Nht-nb.f, T3j.f-nht, ebenfalls ein Denkmal überliefert:

Der Naophor Kairo JE 41301, möglicherweise in Mitrahina gefunden, war bisher aufgrund eines mißverstandenen Epithetons den ${ }^{17}$. Tatsächlich muß er aber aus Naukratis stammen, wie de Meulenaere in einer selbstkritischen Fußnote festgestellt hat ${ }^{18}$. Dieser Naophor (mit einem Verklärungstext auf der Rückseite) gehört einem Mann ohne jeden Titel namens T3j.f-nht ms(.n) Ns-Nbt-hwt; sein Vater heißt

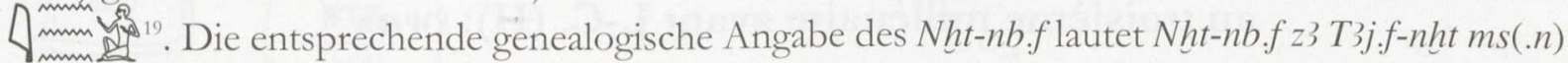
$N s-N b t-h w t$. Normalerweise müßte sich das $m s(. n)$ auf den Besitzer des Denkmals beziehen, also auf Nht-nb.f, so daß Ns-Nbt-hwt seine Mutter wäre. Nicht auszuschließen ist aber, daß es sich in diesem Fall tatsächlich auf den Vater T3j.f-nht bezieht: Auf der erwähnten Statue JE 41301 nennt T3j.f-nht im Haupttext auf dem Rückenpfeiler nur seine Mutter. Es wäre denkbar, daß sie aus einer prominenteren Familie stammte als der Vater und demzufolge der Sohn allgemein als T3j.f-nht, Sohn der Ns-Nbt-hwt bekannt war. Sollte dies der Fall sein und auf beiden Objekten ein T3j.f-nht $m s(. n) N s-N b t$ - $h w t$ genannt sein, dürfte es sich sehr wahrscheinlich um denselben Mann handeln.

Für eine genauere Datierung der „Altarbasis“ wäre dadurch aber nicht viel gewonnen: Dem allgemeinen Eindruck nach sollte sie nicht allzu spät anzusetzen sein. Daressy stützt seinen Vorschlag, sie in die frühe Ptolemäerzeit zu datieren, nur auf den Namen des Besitzers (Nht-nb.f), den er nach dem damaligen Stand der Dinge für identisch mit dem des Königs Nektanebos („II.") hielt, des letzten „einheimischen“ Herrschers ${ }^{20}$. Demzufolge wäre Nht-nb.f unter der Regierung des gleichnamigen Königs geboren worden, sein Denkmal also ca. 1-2 Generationen später anzusetzen, d. h. nach jetzigem Verständnis in die 30. Dynastie, nicht in die frühe Ptolemäerzeit. Aber diese Kalkulation ist natürlich alles andere als sicher. Die Statue JE 41301 ist am oberen Ende des Rückenpfeilers mit einem Relief versehen, in dem eine Person einen stierköpfigen Gott anbetet. Derartige Szenen auf dem Rückenpfeiler sind ab der 30. Dynastie belegt ${ }^{21}$. Sowohl die Statue als auch die Altarbasis könnten also in die 30. Dynastie oder etwas später zu datieren sein.

Daressy, ASAE 17, 1917, 23-4; H. de Meulenaere/P. Mackay, Mendes II, Warminster 1976, p. 199 (67); pl. 26.

Hommages à Jean Leclant, Vol. 4: Varia, BdE 106, 1994, p. 70, n. 42.

Vgl. zu diesem Namen G. Fecht, MDAIK 16, 1958, 113, n. 4.

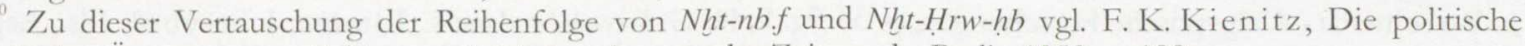
Geschichte Ägyptens vom 7. bis zum 4. Jahrhundert vor der Zeitwende, Berlin 1953, p. 199.

${ }^{21}$ Vgl. Bothmer, Egyptian Sculpture, p. 99: "The later very frequent scene of workship before a single god already occurred in the reign of Nectanebo I." 